\title{
Variational stability and the form of the Schwinger multichannel variational principle
}

\author{
Carl Winstead and Vincent McKoy \\ Noyes Laboratory of Chemical Physics, California Institute of Technology, Pasadena, California 91125
}

(Received 6 March 1992)

\begin{abstract}
We derive the Schwinger multichannel variational principle [K. Takatsuka and V. McKoy, Phys. Rev. A 23, 2352 (1981); 30, 1734 (1984)] by a method that emphasizes the question of variational stability. The present approach is in some respects more direct than the original method and serves to clarify the role of a certain parameter that occurs in the formulation.
\end{abstract}

PACS number(s): $34.10 .+\mathrm{x}, 34.80 . \mathrm{Gs}, 34.80 . \mathrm{Bm}$

\section{INTRODUCTION}

The Schwinger multichannel (SMC) method [1] has proven to be an effective tool for the study of low-energy electron-molecule collisions [2]. Originally the SMC variational functional was derived [1] through the combination of terms arising from a projected LippmannSchwinger equation and terms arising from a projected Schrödinger equation, the latter being introduced on grounds of completeness. This combination yielded a functional that was equivalent to the $T$ matrix but that contained an apparently arbitrary parameter $a$; specifically, when exact wave functions were employed in it, the functional gave the correct $T$-matrix element regardless of the value of $a$ chosen. Additional arguments were then introduced that determined $a$ via the requirement that certain matrix elements vanish, thereby insuring variational stability. However, recently the necessity of this restriction on $a$ has been questioned [3]. The object of this Brief Report is to show how the SMC formulation arises as perhaps the simplest multichannel extension of Schwinger's original expression for the scattering amplitude [4] that is (i) computationally feasible and (ii) variationally stable. Thus a somewhat different route is taken than in earlier work. Rather than introduce a projected Schrödinger equation at the outset, we make the minimal modification to the Schwinger form necessary to satisfy requirement (i), namely projection of the Lippmann-Schwinger equation onto open channels, followed by the minimal additional modification necessary to recover (ii). The same result is obtained as in earlier derivations [1], except that a certain operator occurs in place of the $a$ parameter. No differences in the working equation result, however; in particular, the matrix elements of this operator uniquely and straightforwardly determine $a$. In addition to clarifying aspects of the SMC formulation, the approach taken here can be of general use in developing variational principles from projected equations.

\section{FORMULATION}

As a preliminary step, it is useful to recall the derivation of the original Schwinger variational principle. We begin with the Lippmann-Schwinger equation,

$$
\Psi_{n}^{(+)}=S_{n}+G^{(+)} V \Psi_{n}^{(+)},
$$

where $S_{n}$ is a solution of the interaction-free equation $H_{0} S_{n}=E S_{n}, G^{(+)}$is the Green's function associated with $H_{0}$, and $\Psi_{n}^{(+)}$is a solution to the complete equation $H \Psi_{n}^{(+)}=E \Psi_{n}^{(+)}$, with $H=H_{0}+V ; \quad V$ is the targetscatterer interaction. We multiply Eq. (1) by $V$ to obtain

$$
V S_{n}=V\left(1-G^{(+)} V\right) \Psi_{n}^{(+)},
$$

and then use Eq. (2) in a standard expression for the $T$ matrix element,

$$
T_{m n}=\left\langle\Psi_{m}^{(-)}|V| S_{n}\right\rangle,
$$

to write

$$
T_{m n}=\left\langle\Psi_{m}^{(-)}\left|\boldsymbol{V}\left(1-G^{(+)} V\right)\right| \Psi_{n}^{(+)}\right\rangle .
$$

Finally, we combine Eqs. (3) and (4) with a third form for $T_{m n}$ to obtain an expression that is stable [4] with respect to variation of $\Psi_{m}^{(-)}$and $\Psi_{n}^{(+)}$:

$$
\begin{aligned}
\widetilde{T}_{m n}= & \left\langle S_{m}|\boldsymbol{V}| \Psi_{n}^{(+)}\right\rangle+\left\langle\Psi_{m}^{(-)}|\boldsymbol{V}| S_{n}\right\rangle \\
& -\left\langle\Psi_{m}^{(-)}\left|\boldsymbol{V}\left(1-\boldsymbol{G}^{(+)} \boldsymbol{V}\right)\right| \Psi_{n}^{(+)}\right\rangle .
\end{aligned}
$$

The stability of this form is demonstrable by considering the first-order variations in $\widetilde{T}_{m n}$ that arise from variations $\left\langle\delta \Psi_{m}^{(-)}\right|$and $\left|\delta \Psi_{n}^{(+)}\right\rangle$:

$$
\begin{aligned}
\delta \widetilde{T}_{m n} & =\left\langle\delta \Psi_{m}^{(-)}|V| S_{n}\right\rangle-\left\langle\delta \Psi_{m}^{(-)}\left|V\left(1-G^{(+)} V\right)\right| \Psi_{n}^{(+)}\right\rangle \\
& =0,
\end{aligned}
$$

because of Eq. (2); also

$$
\begin{aligned}
\delta \widetilde{T}_{m n} & =\left\langle S_{m}|\boldsymbol{V}| \delta \Psi_{n}^{(+)}\right\rangle-\left\langle\Psi_{m}^{(-)}\left|\boldsymbol{V}\left(1-\boldsymbol{G}^{(+)} \boldsymbol{V}\right)\right| \delta \Psi_{n}^{(+)}\right\rangle \\
& =\left\langle S_{m}|\boldsymbol{V}| \delta \Psi_{n}^{(+)}\right\rangle-\left\langle\Psi_{m}^{(-)}\left|\left(1-V G^{(+)}\right) \boldsymbol{V}\right| \delta \Psi_{n}^{(+)}\right\rangle \\
& =0,
\end{aligned}
$$

using the adjoint of Eq. (2).

In multichannel, many-particle applications, it is essential for practical reasons to work with a projected Green's function whose representation does not require knowledge of continuum states of the target system [1,5]. We therefore introduce a projector $P_{i}$ onto open channels, whose action on the Green's function will remove the unwanted components:

(c) 1993 The American Physical Society 


$$
\begin{aligned}
P_{i}=\sum_{l \in \text { open }} \mid & \left|\Phi_{l}(1,2, \ldots, i-1, i+1, \ldots, N+1)\right\rangle \\
& \times\left\langle\Phi_{l}(1,2, \ldots, i-1, i+1, \ldots, N+1)\right| .
\end{aligned}
$$

$\Phi_{l}$ is an $N$-electron function, one of the open-channel target states (ionization channels are assumed closed). Operating on Eq. (1) with $P_{i}$, we obtain

$$
P_{i} \Psi_{n}^{(+)}=S_{i n}+G_{P i}^{(+)} V_{i} \Psi_{n}^{(+)}
$$

where $G_{P i}^{(+)}=P G_{i}^{(+)}$and we have used the fact that $P_{i} S_{i n}=S_{i n}$. In Eq. (6) and the following, we have introduced the subscript $i$ on $S_{n}, V$, and $G^{(+)}$, as well as on $P$, as a reminder that these entities correspond to a particular partitioning of the Hamiltonian into zeroth-order and interaction pieces, $H_{0 i}$ and $V_{i}$, that picks out electron $i$ as the scattering electron (usually $[1,2]$ we take $i=N+1$, although the choice is arbitrary). Thus, for example,

$$
V_{i}=-\sum_{\alpha} \frac{Z_{\alpha}}{\left|\mathbf{R}_{\alpha}-\mathbf{r}_{i}\right|}+\sum_{j(\neq i)}^{N+1} \frac{1}{\left|\mathbf{r}_{j}-\mathbf{r}_{i}\right|}
$$

where $\alpha$ labels the nuclei.

Equation (6) yields a new expression for the $T$ matrix,

$$
T_{m n}=\left\langle\Psi_{m}^{(-)}\left|V_{i} P_{i}\left(1-G_{i}^{(+)} V_{i}\right)\right| \Psi_{n}^{(+)}\right\rangle,
$$

that we can use instead of Eq. (4) to try to construct a variational functional analogous to the Schwinger functional:

$$
\begin{aligned}
\widetilde{T}_{m n}^{\prime}= & \left\langle S_{i m}\left|V_{i}\right| \Psi_{n}^{(+)}\right\rangle+\left\langle\Psi_{m}^{(-)}\left|V_{i}\right| S_{i n}\right\rangle \\
& -\left\langle\Psi_{m}^{(-)}\left|V_{i} P_{i}\left(1-G_{i}^{(+)} V_{i}\right)\right| \Psi_{n}^{(+)}\right\rangle .
\end{aligned}
$$

Not surprisingly, this naive attempt can readily be shown to fail. On taking variations in $\Psi_{m}^{(-)}$, we find

$$
\begin{aligned}
\delta \widetilde{T}_{m n}^{\prime}= & \left\langle\delta \Psi_{m}^{(-)}\left|V_{i}\right| S_{i n}\right\rangle \\
& -\left\langle\delta \Psi_{m}^{(-)}\left|V_{i} P_{i}\left(1-G_{i}^{(+)} V_{i}\right)\right| \Psi_{n}^{(+)}\right\rangle \\
= & 0,
\end{aligned}
$$

which follows from Eq. (2) and $P_{i} S_{i n}=S_{i n}$. This is as desired. However, on varying $\Psi_{n}^{(+)}$, we find

$$
\begin{aligned}
\delta \widetilde{T}_{m n}^{\prime} & =\left\langle S_{i m}\left|V_{i}\right| \delta \Psi_{n}^{(+)}\right\rangle-\left\langle\Psi_{m}^{(-)}\left|V_{i} P_{i}\left(1-G_{i}^{(+)} V_{i}\right)\right| \delta \Psi_{n}^{(+)}\right\rangle \\
& =\left\langle S_{i m}\left|V_{i}\right| \delta \Psi_{n}^{(+)}\right\rangle-\left\langle\Psi_{m}^{(-)}\left|\left(1-V_{i} G_{i}^{(+)}\right) P_{i} V_{i}\right| \delta \Psi_{n}^{(+)}\right\rangle+\left\langle\Psi_{m}^{(-)}\left|P_{i} V_{i}-V_{i} P_{i}\right| \delta \Psi_{n}^{(+)}\right\rangle \\
& =\left\langle\Psi_{m}^{(-)}\left|P_{i} V_{i}-V_{i} P_{i}\right| \delta \Psi_{n}^{(+)}\right\rangle \\
& \neq 0
\end{aligned}
$$

The functional of Eq. (7) is thus not variationally stable.

To recover stability, we clearly must add a term to Eq. (7) that will not destroy the stability with respect to variations $\delta \Psi_{m}^{(-)}$that already exist, but will cancel the $\left\langle\Psi_{m}^{(-)}\left|P_{i} V_{i}-V_{i} P_{i}\right| \delta \Psi_{n}^{(+)}\right\rangle$matrix element left over on varying $\Psi_{n}^{(+)}$. Of course, the resulting expression must also continue to represent the $T$ matrix. These rather strict requirements suggest that we consider adding a term of the form $\left\langle\Psi_{m}^{(-)}|Q \hat{H}| \Psi_{n}^{(+)}\right\rangle$, where $\hat{H}=E-H$ and $Q$ is to be determined. While we have not established that this form is the only possible choice, no other possibility suggests itself. Since $\hat{H} \Psi_{n}^{(+)}=0$, the value of the expression for $T_{m n}$ is unchanged, and stability with respect to variations $\delta \Psi_{m}^{(-)}$is preserved. At the same time, the form of $Q$ may be manipulated to achieve the desired stability with respect to variations $\delta \Psi_{n}^{(+)}$.

An obvious way to eliminate the unwanted $\left\langle\Psi_{m}^{(-)}\left|P_{i} V_{i}-V_{i} P_{i}\right| \delta \Psi_{n}^{(+)}\right\rangle$term is for $Q \hat{H}$ to combine with it to yield an expression in which $\hat{H}$ is on the left and a function that vanishes asymptotically in all the electron coordinates is on the right. In that case we will be able to write the resulting matrix element as the Hermitian conjugate of an expression in which $\hat{H}$ operates to the right on $\left|\Psi_{m}^{(-)}\right\rangle$, and we then can make use of $\hat{H} \Psi_{m}^{(-)}=0$ to show that the matrix element vanishes. Consider therefore $Q=P_{i}-R_{i}$, where $R_{i}$ is an operator that selects from a fully antisymmetric $(N+1)$-electron wave function a piece that corresponds to electron $i$ as the scattering electron and the remaining electrons on the target. [For the closed channels, such a partitioning is not unique. As an example of how to define such an $R_{i}$, we note that any $(N+1)$-electron function with the specified asymptotic behavior may be represented, though not uniquely, in a basis set of the form

$$
\begin{aligned}
& \Xi_{j}\left(\mathbf{r}_{1}, \mathbf{r}_{2}, \ldots, \mathbf{r}_{N+1}\right) \\
& \quad=A_{i}\left[\Phi_{j}\left(\mathbf{r}_{1}, \mathbf{r}_{2}, \ldots, \mathbf{r}_{i-1}, \mathbf{r}_{i+1}, \ldots, \mathbf{r}_{N+1}\right) \xi_{j}\left(\mathbf{r}_{i}\right)\right],
\end{aligned}
$$

where $A_{i}$ is an antisymmetrizer between electron $i$ and the remaining electrons, and the (antisymmetric) $\mathrm{N}$ electron function $\Phi_{j}$ vanishes asymptotically in all coordinates, but $\zeta_{j}\left(\mathbf{r}_{i}\right)$ does not necessarily do so. An appropriate $R_{i}$ associated with this expansion simply replaces each $\Xi_{j}$ with the nonantisymmetric function $\left.\Phi_{j} \zeta_{j}.\right] \quad R_{i}$ has the same effect as $P_{i}$ on the open-channel portion of the wave function, but it does not annihilate the closed-channel part as $P_{i}$ does. In effect, $R_{i}$ merely removes the antisymmetrization between electron $i$ and the remaining electrons, and therefore $R_{i}$ commutes with $H$, a property we will need later. We will also use the fact that $P_{i}$ commutes with the interaction-free Hamiltonian $H_{0 i}$.

Using the assumed form for $Q$ and these commutation properties, some very straightforward rearrangement shows that

$$
\begin{aligned}
\left\langle\Psi_{m}^{(-)}\right| & Q \hat{H}+P_{i} V_{i}-V_{i} P_{i}\left|\delta \Psi_{n}^{(+)}\right\rangle \\
& =\left\langle\Psi_{m}^{(-)}\left|\hat{H}_{0 i} P_{i}-P_{i} V_{i}-\hat{H} R_{i}+P_{i} V_{i}-V_{i} P_{i}\right| \delta \Psi_{n}^{(+)}\right\rangle \\
& =\left\langle\Psi_{m}^{(-)}|\hat{H} Q| \delta \Psi_{n}^{(+)}\right\rangle,
\end{aligned}
$$


where $\hat{H}_{0 i}=E-H_{0 i}$. The second line above may be replaced with $\left\langle\delta \Psi_{n}^{(+)}|Q \hat{H}| \Psi_{m}^{(-)}\right\rangle^{*}=0$, provided that the function $Q \delta \Psi_{n}^{(+)}$vanishes asymptotically (so that no surface terms arise in doing the integrations by parts that are necessary to "turn around" the kinetic-energy operators contained in $\hat{H}) . R_{i} \delta \Psi_{n}^{(+)}$and $P_{i} \delta \Psi_{n}^{(+)}$both vanish as $r_{j} \rightarrow \infty$ for all $j$ not equal to $i$, since we are assuming that the total energy is below the ionization threshold, and since the variation $\delta \Psi_{n}^{(+)}$must have the same asymptotic form as $\Psi_{n}^{(+)}$. Thus, we need only worry about $r_{i} \rightarrow \infty$, but in this case $Q \delta \Psi_{n}^{(+)}$also vanishes asymptotically, because the $R_{i} \delta \Psi_{n}^{(+)}$and $P_{i} \delta \Psi_{n}^{(+)}$terms cancel. This establishes stability with respect to variations $\delta \Psi_{n}^{(+)}$.

It remains to note that, in doing calculations, we can replace $R_{i}$ with $1 /(N+1)$, since

$$
\left\langle\chi_{s}\left|R_{i} \hat{H}\right| \chi_{t}\right\rangle=\frac{1}{N+1}\left\langle\chi_{s}|\hat{H}| \chi_{t}\right\rangle
$$

for any two fully antisymmetric $(N+1)$-electron functions $\chi_{s}$ and $\chi_{t}$, and these are the only types of terms in $R_{i}$ that arise in the SMC expression. We should also stress that it is essential to use $Q=P_{i}-R_{i}$, and not just $Q=P_{i}-1 /(N+1)$, since otherwise $Q \delta \Psi_{n}^{(+)}$will not vanish asymptotically as $r_{j}$ goes to infinity in the case $j \neq i$. This is somewhat in contrast to the point of view taken in previous derivations [1], where a scalar parameter $a$ occurred in place of the operator $R_{i}$.

Finally, we summarize our variationally stable expression - the naive form, Eq. (7), plus the $Q \hat{H}$ correction term:

$$
\begin{aligned}
\widetilde{T}_{m n}^{\prime \prime} & =\left\langle S_{i m}\left|V_{i}\right| \Psi_{n}^{(+)}\right\rangle+\left\langle\Psi_{m}^{(-)}\left|V_{i}\right| S_{i n}\right\rangle-\left\langle\Psi_{m}^{(-)}\left|V_{i} P_{i}\left(1-G_{i}^{(+)} V_{i}\right)\right| \Psi_{n}^{(+)}\right\rangle+\left\langle\Psi_{m}^{(-)}\left|\left(P_{i}-R_{i}\right) \hat{H}\right| \Psi_{n}^{(+)}\right\rangle \\
& =\left\langle S_{i m}\left|V_{i}\right| \Psi_{n}^{(+)}\right\rangle+\left\langle\Psi_{m}^{(-)}\left|V_{i}\right| S_{i n}\right\rangle-\left\langle\Psi_{m}^{(-)}|| \frac{1}{N+1}-P_{i}\right] \hat{H}+V_{i} P_{i}-V_{i} G_{P_{i}}^{(+)} V_{i}\left|\Psi_{n}^{(+)}\right\rangle
\end{aligned}
$$

In the second line of Eq. (8), we have replaced $R_{i}$ with $1 /(N+1)$, which, again, is allowable only in matrix elements, to show that the form reduces to precisely the usual SMC expression [1].

\section{SUMMARY}

We have shown how the SMC expression for the scattering amplitude may be derived by imposing the requirement of variational stability on a functional that is initially of the Schwinger form except for the use of a projected Lippmann-Schwinger equation. In contrast to previous derivations [1], the parameter $a$ is shown to be uniquely determined as, in essence, the matrix element of a certain operator whose form is dictated by the stability requirement. The present approach thus clarifies the relationship of the SMC functional to the Schwinger functional and provides insight into the connection between its form and the fundamental question of stability. A similar approach can be used in modifying other variational principles when practical considerations suggest the use of projected equations in their implementation.

\section{ACKNOWLEDGMENT}

This work was supported by the National Science Foundation under Grant No. PHY-9021933.
[1] K. Takatsuka and V. McKoy, Phys. Rev. A 23, 2352 (1981); 30, 1734 (1984); M. A. P. Lima and V. McKoy, ibid. 38, 501 (1988).

[2] See, for example, M. A. P. Lima, K. Watari, and V. McKoy, Phys. Rev. A 39, 4312 (1989); H. P. Pritchard, V. McKoy, and M. A. P. Lima, ibid. 41, 546 (1990); C. Winstead, P. G. Hipes, M. A. P. Lima, and V. McKoy, J.
Chem. Phys. 94, 5455 (1991); Q. Sun, C. Winstead, M. A. P. Lima, and V. McKoy, ibid. 96, 3531 (1992).

[3] W. M. Huo and C. A. Weatherford, Bull. Am. Phys. Soc. 36, 1265 (1991).

[4] J. Schwinger, Phys. Rev. 72, 742 (1947).

[5] S. Geltman, Topics in Atomic Collision Theory (Academic, New York, 1969), pp. 95ff. 